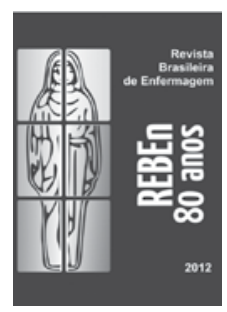

\title{
Contribuição das linguagens padronizadas para a produção do conhecimento, raciocínio clínico e prática clínica da Enfermagem
}

\author{
Contribution of standardized languages for knowledge production, \\ clinical reasoning and clinical Nursing practice \\ Contribución de los lenguajes estandarizados para la producción del conocimiento, \\ razonamiento clínico y práctica clínica de Enfermería
}

\section{Emilia Campos de Carvalho', Dina de Almeida Lopes Monteiro da Cruz", T. Heather Herdman"I \\ ' Universidade de São Paulo, Escola de Enfermagem de Ribeirão Preto, Departamento de Enfermagem Geral e Especializada. Ribeirão Preto-SP, Brasil. \\ "Universidade de São Paulo, Escola de Enfermagem, \\ Departamento de Enfermagem Médico-Cirúrgica. São Paulo-SP, Brasil. \\ II' Universidade de Wisconsin, Escola de Enfermagem. NANDA Internacional, Inc. Green Bay, Wisconsin, Estados Unidos.}

Submissão: 19-08-2013 Aprovação: 19-08-2013

\section{RESUMO}

Os sistemas de linguagens padronizadas são instrumentos importantes para lidar com a crescente complexidade do cuidado de enfermagem. Neste artigo os autores apresentam os principais benefícios que o uso desses sistemas oferece para o raciocínio clínico requerido no cuidado de enfermagem, para a construção e organização do conhecimento da disciplina e para a prática clínica de enfermagem. As potenciais contribuições dos sistemas de linguagens padronizadas nesses campos derivam do fato de tais sistemas oferecerem estrutura formal para apoiar o raciocínio clínico, organizar o conhecimento e a experiência de enfermagem.

Descritores: Classificação; Julgamento; Competência Clínica; Prática Profissional; Conhecimento.

\begin{abstract}
The standardized language systems are important tools for dealing with the increasing complexity of nursing care. In this article the authors present the main benefits that the use of these systems provide for the required clinical reasoning in nursing care, the construction and organization of knowledge of the discipline, and for the clinical practice of nursing. The potential contributions of the standardized language systems in these fields stem from the fact that these systems provide a formal structure for supporting clinical reasoning, organizing knowledge and nursing experience.
\end{abstract}

Key words: Classification; Judgment; Clinical Competency; Professional Practice; Knowledge.

\section{RESUMEN}

Los sistemas de lenguajes estandarizados son herramientas importantes para hacer frente a la creciente complejidad de los cuidados de enfermería. En este artículo los autores presentan los principales beneficios que el uso de estos sistemas ofrece para el razonamiento clínico requerido en los cuidados de enfermería, para la construcción y organización del conocimiento de la disciplina, y para la práctica clínica de la enfermería. Las posibles contribuciones de los sistemas de lenguajes estandarizados en estos ámbitos se derivan del hecho de que estos sistemas proporcionan una estructura formal para apoyar el raciocinio clínico, organizar la experiencia y los conocimientos de enfermería.

Palabras clave: Clasificación; Juicio; Competencia Clínica; Práctica Profesional; Conocimiento. 


\section{INTRODUÇÃO}

A Enfermagem constitui-se atualmente em uma área do saber útil à sociedade, utilidade esta traduzida essencialmente pelo desenvolvimento de um conjunto de atividades que são essenciais à vida dessa sociedade, mas ainda não reconhecida como fazendo parte de um campo autônomo de saber e de intervenção(1).

Essa afirmação, apresentada no contexto da discussão da transição do ensino na enfermagem portuguesa, pode ser expandida para nosso meio; tal realidade pressupõe, no dizer do autor, que se deva olhar para além de seus significados expressos e buscar as razões da sua origem e as formas de muda-las. Dentre as dimensões consideráveis - o cenário, os atores e os saberes constituídos e divulgados - destacamos um olhar para o conhecimento produzido. A forma como uma disciplina/profissão se comunica com a sociedade, como seus profissionais se comunicam entre si ou com outros membros de sua área, ou ainda como cada profissional lança mão de seu conhecimento para expressar a sua pratica ou ensinar os seus futuros colegas, passa pela questão de como o conhecimento da profissão está organizado.

Este artigo trata dos argumentos que apoiam o pressuposto de que o uso de sistemas de linguagens padronizadas é fundamental para o desenvolvimento da enfermagem como disciplina e profissão. Serão tratados os aspectos relacionados à produção e ao desenvolvimento do conhecimento, ao raciocínio clínico e à prática clínica de enfermagem. A expressão sistemas de linguagens padronizadas de enfermagem (SLP) refere-se a estruturas que organizam uma terminologia acordada entre os profissionais enfermeiros para descrever as avaliações, intervenções e resultados pertinentes ao cuidado de enfermagem ${ }^{(2)}$. As unidades singulares de um SLP são os termos ou expressões que veiculam os conceitos pertinentes à área a que o sistema se aplica.

\section{CONCEITOS E CONHECIMENTO}

A elucidação da linguagem é o modo mais efetivo de definir a essência de um conceito e de solucionar problemas relevantes de pesquisas, como especificar a estrutura lógica de uma dada expressão, de significados comuns de termos, explicitações centrais para reduzir limitações, ambiguidades e inconsistências do uso ordinário para propor uma reinterpretação para aumentar a claridade e precisão dos significados.

Conceito é a instância mediadora entre o sujeito e o real; é uma ideia ou uma abstração de um evento concreto $^{(3)}$ ou a unidade de pensamento( ${ }^{(4)}$. É, portanto, uma representação simbólica das dimensões de uma realidade/fenômeno. Conceitos podem também ser entendidos como rótulos que descrevem fenômenos e fornecem uma síntese concisa de pensamentos ${ }^{(5-6)}$.

Cada ciência usa seus próprios conceitos para a comunicação de seus conhecimentos ${ }^{(7)}$. Entende-se por conhecimento o resultado da cognição. À medida que aumenta o conhecimento de determinada matéria, muda a nossa concepção dessa matéria; quando os nossos conceitos se tornam mais adequados, podemos aprender cada vez mais.

\section{SISTEMAS DE LINGUAGENS PADRONIZADAS E PRO- DUÇÃO DE CONHECIMENTO}

Para ser efetivo, o uso da linguagem requer a precisão de conceitos. No âmbito da enfermagem, um dos fatores que contribuem para os vazios de um corpo de conhecimento bem definido é a falta de conceitos clarificados. Como componentes da base de conhecimentos da enfermagem, os conceitos, empregados nas teorias e na prática de enfermagem, são de dois tipos: os relacionados ao conhecimento abstrato (Ex. bem estar) e os orientados para solucionar as questões de enfermagem (Ex. condicionamento cardiovascular) ${ }^{(3)}$.

Portanto, justifica-se a preocupação de diversos autores ao questionarem se os conceitos usados na enfermagem são bem definidos, se são identificados de forma inequívoca, se são todos conhecidos e se apresentam os elementos que os caracterizam, as condições em que ocorrem, suas consequências e como devem ser mensurados.

\section{Os conceitos nas linguagens padronizadas}

Todo SLP trata da organização de conceitos. Na enfermagem há diversos SLP que abrigam os conceitos relevantes para a prática clínica. Apesar dos acalorados debates quanto a supremacia de um ou outro sistema, o que nos interessa aqui é o olhar sobre 'as coisas' que são organizadas dentro desses sistemas, e como a existência de um sistema que organize essas 'coisas' pode influenciar o desenvolvimento do conhecimento e da prática clínica.

As 'coisas' que são organizadas dentro dos SLP são termos ou expressões que representam conceitos relevantes para a disciplina da enfermagem. Considerando os metaparadigmas da enfermagem, que são a pessoa, o ambiente, a enfermagem e a saúde ${ }^{(8)}$, os conceitos relevantes são aqueles que dizem respeito à interação da pessoa com o ambiente e que podem ser influenciados pela enfermagem em direção à melhor saúde possível. Poderíamos dizer, de outra forma, que os conceitos relevantes para a enfermagem são as respostas das pessoas às circunstâncias de vida e saúde (diagnósticos); as ações que a enfermagem realiza para influenciar positivamente essas respostas (intervenções) e obter ou manter as melhores condições possíveis de saúde e bem-estar (resultados). Dessas afirmações podemos concluir que os SLP organizam conceitos relativos a diagnósticos, intervenções e resultados de enfermagem.

Os SLP que oferecem estrutura para organizar diagnósticos, intervenções e resultados de enfermagem são instrumentos importantes para lidar com a crescente complexidade da enfermagem no que diz respeito à produção de conhecimento, ao raciocínio clínico e à prática clínica.

Espera-se que os conceitos empregados no interior da própria enfermagem para determinar uma dada situação sejam equivalentes tanto em distintas regiões geográficas como dentro das instituições em que a enfermagem atua. A literatura aponta a preocupação de se empregar na enfermagem conceitos que não apresentem erros ou divergências, que sejam 
identificáveis, mensuráveis ou passíveis de avaliação, que tenham seus componentes conhecidos e que apontem tendências de suas consequências ou da sua presença ${ }^{(9)}$.

O desenvolvimento de qualquer ciência está relacionado à existência de uma forma organizada de seu vocabulário, ou seja, de um SLP, que nada mais é que um conjunto de conceitos organizados da forma mais lógica possível. A organização de conceitos pode ser auxiliada pela ontologia, ciência que estuda a natureza de uma entidade de interesse. Ela serve para criar terminologias, codificar o conhecimento ou representações formais para que possa ser compartilhado. Mas, de nada adianta se não houver um bom conteúdo sobre o que se deseja organizar.

De acordo com a literatura, há várias concepções para ontologia que, em sua essência, do ponto de vista da filosofia, trata do estudo da existência. Tem sido considerada como representação de um conceito entre os membros uma dada comunidade; também é aceita como a especificação formal (representação) explícita de uma conceitualização compartiIhada(10). "É uma teoria lógica para relacionar o significado pretendido de um vocabulário formal, isto é, seu comprometimento com uma conceitualização particular de mundo"(11).

A ontologia pode ser aplicada em diferentes áreas, com propósitos de definir e criar relação entre objetos, a partir de linguagem com semântica bem definida como, por exemplo, a que busca prover auxílio na realização de tarefas diárias de pessoal de saúde em ambiente hospitalar ${ }^{(12)}$. As ontologias servem para criar terminologias únicas, de modo que o conhecimento possa ser compartilhado e reutilizado.

A organização do conhecimento de enfermagem e a sua representação tornam-se necessários, dada a sua abrangência, para a compreensão de sua prática, entendendo-se nela o ensino, a assistência e a pesquisa.

Os elementos básicos de uma ontologia são: as classes, ou seja, as entidades organizadas em sistemas de linguagem padronizada; as relações ou interações entre os conceitos de um domínio; os axiomas, usados para constituir as sentenças sempre verdadeiras; e as instâncias, que representam os elementos específicos ou os próprios dados ${ }^{(10)}$.

Os SLP organizam as informações de mais genéricas a mais especificas, utilizando relação hierárquica ou de gênero-espécie entre os termos ${ }^{(13)}$. Além de classificar e facilitar acesso a informação, visam, dentre outros fins, representar conceitos, controlar significados distintos, favorecer a comunicação entre especialistas e outros públicos ${ }^{(14)}$. Uma taxonomia, que é um tipo de SLP, deve ter regras rigorosas para a categorização de seus elementos. Para ser válido, um SLP não deve ter grandes lacunas; contudo, deve ser dinâmico no sentido de permitir que novos conceitos, fruto da ampliação do conhecimento, sejam incorporados.

A representação e organização do conhecimento possibilitam conceituar fenômenos relevantes para a profissão, clarificar esses fenômenos e prover a base de conhecimento para o profissional ter a compreensão sobre tal fenômeno ${ }^{(15)}$. A seleção de conceito pertinente, isto é, que representa o fenômeno adequadamente, contribui para a segurança do paciente, pois reduz informações dúbias e indevidas sobre o fenômeno e permite eleger intervenções adequadas.
Os SLP, portanto, são fundamentais para desenvolver o conhecimento da enfermagem ao mesmo tempo em que dependem fundamentalmente do conhecimento existente. O estudo dos conceitos contidos nos SLP fornecem resultados indispensáveis para a produção e desenvolvimento do conhecimento de enfermagem. Cada termo ou expressão dentro de um SLP precisa ser identificado, analisado, clarificado e desenvolvido para que possa representar de forma válida uma unidade de conhecimento da disciplina.

A área de estudo de conceitos envolve processos de raciocínio dedutivo, indutivo ou de ambos, com regras estabelecidas, para identificar as características do conceito em questão, o seu emprego, a sua representatividade e as suas relações com outros conceitos.

Um conceito pode ser empregado por diferentes disciplinas. Por exemplo, o conceito depressão(16); ainda que esse termo tenha um significado "para baixo", quando empregado nos diferentes contextos da meteorologia, economia e psicologia, congrega características peculiares ${ }^{(16)}$. Por outro lado, um fenômeno pode ter vários conceitos que se referem a ele $\mathrm{e}^{(17)}$; o fenômeno dor, por exemplo, pode agregar os conceitos de dor aguda, dor crônica ou dor de parto (cada um representando um conceito e seus respectivos atributos específicos ou suas características).

O movimento para clarificar os conceitos que contribuem para a produção do conhecimento e para promover a unificação da linguagem empregada por enfermeiros não é recente. Em um artigo sobre a revisão histórica dos métodos de validação de diagnóstico foi apontada a contribuição de vários autores, desde o inicio dos anos 1990, com pesquisas de desenvolvimento de conceitos e a importância da análise de conceito como fase fundamental da pesquisa de validação de um diagnóstico especifico ${ }^{(18)}$.

Os esforços da profissão em definir claramente seus conceitos de interesse e em descrever e medir os fenômenos da prática em enfermagem já foram bem documentados ${ }^{(19)}$. Alguns referenciais que dão sustentação às investigações nessa área e alguns exemplos da produção nacional empregando tais modelos teóricos/metodológicos podem ser exemplificados.

Modelo clássico de estudo de conceito ${ }^{(19)}$ já foi empregado em diversos estudos brasileiros, como por exemplo, para construir e elucidar conceitos como os de fadiga ${ }^{(20)}$; adesão ao tratamento anti-hipertensivo ${ }^{(21)}$; risco de débito cardíaco diminuído ${ }^{(22)}$,e recuperação cirúrgica retardada ${ }^{(23)}$. Outro modelo $^{(24)}$ para desenvolver e refinar conceitos, foi aplicado, por exemplo, para a construção do conceito recuperação cirúrgica retardada ${ }^{(25)}$. Outras abordagens também foram empregadas para o estudo de conceitos como a construção do conceito vulnerabilidade da família ${ }^{(26)}$ ou o de morte digna ${ }^{(27)}$.

Apesar da clara ligação dos estudos de conceitos com os SLP, nem sempre os pesquisadores os colocam nesse contexto. Um dos desafios para os pesquisadores da enfermagem é conceber os SLP não só como fontes de conceitos a serem desenvolvidos e refinados, mas também como repositórios legítimos para abrigar os conceitos que são criados pela investigação. Em termos de produção de conhecimento, os SLP são repositórios e fontes de temas de investigação. 
Um SLP de diagnósticos de enfermagem, por exemplo, organiza termos e expressões que representam conceitos sobre respostas humanas ou problema de um paciente ${ }^{(28)}$. Um SLP de resultados contém rótulos representando conceitos que retratam estados, comportamentos ou percepções de um individuo família ou comunidade, medidos ao longo de um contínuo em resposta a uma intervenção, que podem ser avaliados como positivos ou negativos ${ }^{(29)}$. Cada resultado possui uma definição, pelo menos uma escala de medida e uma lista de indicadores relacionados ao conceito de interesse ${ }^{(29)}$. SLP de intervenções, por sua vez, descrevem conceitos relacionados aos comportamentos do enfermeiro. Na Classificação de Intervenções de Enfermagem ${ }^{(30)}$, por exemplo, uma intervenção é qualquer tratamento baseado no julgamento e conhecimento, que seja realizado por enfermeiro, para melhorar um resultado do paciente/cliente. Esse sistema de linguagem padronizada de intervenções oferece, além do titulo ou rótulo (o próprio conceito), uma definição e um conjunto de atividades pertinente ao conceito expresso pelo rótulo.

Para que um SLP continue alcançando seus objetivos e contribuindo com os profissionais em sua prática clínica, é necessário o apoio de pesquisas bem conduzidas, que retratem o conhecimento da área e permitam a inclusão ou reformulação desses conceitos, à luz das mudanças desta prática.

\section{As linguagens padronizadas e o raciocínio clínico}

No âmbito do raciocínio clínico o uso de um SLP fornece estrutura para organizar o conhecimento da disciplina de enfermagem o que é indispensável para o processo de interpretação acurada e útil das observações sobre os pacientes e seus ambientes.

Raciocínio clínico refere-se aos processos cognitivos que os clínicos usam para coletar informações do paciente, analisar os dados, gerar hipóteses e avaliar essas hipóteses. Esses processos permitem que o clínico se mova a partir de dados de avaliação, indicando sinais anormais, sintomas, estados de risco e/ou pontos fortes do paciente para hipóteses sobre a natureza do problema, ou sobre oportunidades de promoção de saúde. Por exemplo, a hipotermia neonatal pode ser causada por prematuridade, exposição do recém-nascido ao meio ambiente frio, ou insuficiência de gordura marrom. Algumas dessas etiologias são modificáveis pelo enfermeiro (por exemplo, o ambiente frio), ao passo que outras são importantes conhecer, mas não podem ser modificadas (por exemplo, a prematuridade). Os enfermeiros não precisam somente de acurácia na avaliação dos dados clínicos; muitas vezes, além de decisões acuradas, é necessário processar as informações de forma rápida e reavaliar quando ocorrem mudanças no estado do paciente. Finalmente, eles precisam de uma forma clara e concisa para comunicar os seus achados e suas conclusões sobre os principais problemas/pontos fortes que o paciente está enfrentando (diagnósticos de enfermagem), os resultados desejáveis para os pacientes e as intervenções de enfermagem selecionadas para alcançar os resultados desejáveis.

Apesar de uma ampla discussão sobre o raciocínio clínico poder ser o mesmo em várias disciplinas clínicas, existem características específicas do raciocínio clínico em cada disciplina. A diferença fundamental é que cada profissional usa o raciocínio clínico com base na sua própria área de atuação - os conceitos, teorias e modelos que representam o conhecimento da disciplina. Assim, o raciocínio clínico do enfermeiro deve ser conduzido a partir de uma perspectiva de enfermagem.

Essa discussão nos leva à pergunta: qual é o conhecimento da disciplina de enfermagem? O conhecimento da disciplina é expresso pelos fenômenos de seu interesse, que foram definidos como: respostas humanas reais ou potenciais de pacientes, famílias, grupos e comunidades para os problemas de saúde e/ ou processos de vida ${ }^{(31)}$. Em última análise, os fenômenos de interesse incluem a resposta humana da pessoa (paciente, família, grupo ou comunidade), o impacto do ambiente sobre aquele que recebe os cuidados de saúde (temperatura ambiente, ruído, som, segurança, poluição, qualidade da água, etc.) e a saúde do paciente / família / grupo / comunidade (estados de risco, os problemas reais, as oportunidades de promoção da saúde). Em outras palavras, o conhecimento da disciplina de enfermagem é o conhecimento dos conceitos que fundamentam os diagnósticos de enfermagem que os enfermeiros identificam, tratam e em relação aos quais eles têm a responsabilidade pelos resultados. Esses diagnósticos são, portanto, referência para orientar as intervenções de enfermagem e para medir os resultados do paciente sensíveis ao cuidado de enfermagem.

Estudos dos anos $1980^{(32)}$ com médicos e estudantes de medicina mostraram que a interpretação clínica depende do reconhecimento pelo clínico daqueles itens de informação que Ihe são pessoalmente relevantes, chamados de 'forceful features', que vamos traduzir como 'características críticas'. Nesse modelo, chamado de Diagnostic Process Thinking, a decisão clínica é descrita pela identificação dos processos cognitivos que ocorrem a medida que o profissional resolve um problema que tem início com a percepção, pelo clínico, de uma questão diagnóstica(33). Isto é, o processo é ativado por questões como "qual é a situação problemática?" ou "qual é a situação que requer intervenção profissional?". Nesse modelo, a percepção de uma questão diagnóstica depende da forma como o conhecimento é estruturado na memória do profissional e ambos, conhecimento e estrutura, variam de pessoa para pes$\mathrm{soa}^{(33)}$. Com base em resultados de estudos clássicos na área ${ }^{(34)}$, admite-se que as diferenças na qualidade das decisões clínicas dependem principalmente das diferenças no conteúdo e estrutura de organização do conhecimento na memória.

As explanações oferecidas pelo modelo do Diagnostic Process Thinking ${ }^{(33)}$ são fundamentos para afirmar que a disponibilidade dos SLP para as decisões clínicas na enfermagem são importantes para a qualidade dessas mesmas decisões. Diante de um SLP de diagnósticos, por exemplo, o enfermeiro dispõe de uma estrutura que lhe permite organizar um universo de possibilidades de situações clínicas que requerem cuidados de enfermagem. Essa estrutura orienta o armazenamento do conhecimento que o profissional vai adquirindo com a experiência e, muito provavelmente, a atribuição de significado às observações em que se fundamentam as interpretações e decisões clínicas que faz. O seguinte excerto ${ }^{(35)}$ bem sintetiza as possíveis contribuições de um SLP para o raciocínio clínico: 
[Um sistema de linguagem padronizada] "... auxilia no julgamento clínico porque representa um "mapa do território" da enfermagem clínica; contém termos que permitem [ao enfermeiro] distanciar-se da experiência imediata, elaborando teoricamente sua atividade prática. ... [pois é] "no processo de criação de significados que os sujeitos organizam sua experiência no mundo, seu conhecimento sobre ele e onde ocorrem as trocas entre os sujeitos"(36).

\section{Ainda da mesma fonte ${ }^{(35)}$}

"Ao usar uma classificação, os enfermeiros vão criando significados para os termos e vão desenvolvendo esses significados. Isso poderá ajuda-los a organizar suas experiências no mundo da enfermagem, seu conhecimento sobre esse mundo. Nessa criação e desenvolvimento, indubitavelmente ocorrem as trocas entre os sujeitos; entre os enfermeiros, entre os enfermeiros e os pacientes e toda a comunidade. Sem um acordo sobre quais sejam os focos clínicos, cada enfermeiro vai delineando um universo particular de possibilidades que tende a ser mais limitado do que o que é oferecido por um sistema de classificação."

O desenvolvimento do conhecimento sobre os conceitos fundamentais para a disciplina de enfermagem contidos nos SLP é um passo essencial para o raciocínio clínico do enfermeiro.

\section{AS LINGUAGENS PADRONIZADAS NA PRÁTICA CLÍNICA}

O uso dos SLP na prática clínica - e aqui prática clínica é entendida como a prática que se dá no âmbito das interações entre as pessoas envolvidas no cuidado; entre os que estão a cuidar e os que estão a ser cuidados - pode contribuir para melhores resultados do cuidado que se oferece. A possibilidade de melhores resultados atribuíveis ao uso de SLP depende, obviamente, da forma como eles são aplicados. Decisões exclusivamente burocráticas para adotar um SLP muito provavelmente pouco ou nada contribuirão. Por outro lado, se os SLP forem adotados como instrumentos auxiliares ao raciocínio clínico, junto com uma compreensão clara da natureza do foco da prática clínica de enfermagem, as contribuições serão viabilizadas.

Os SLP na prática clínica podem ter contribuições especiais no âmbito da avaliação de enfermagem, no âmbito da comunicação e no âmbito do gerenciamento do cuidado.

\section{Linguagens padronizadas e avaliação}

As contribuições dos SLP no âmbito da avaliação de enfermagem são derivadas das contribuições ao raciocínio clínico. A avaliação é a pedra angular do raciocínio clínico e do cuidado de enfermagem, pois sem avaliação não há dados a partir dos quais inferir possíveis diagnósticos, sem os quais não é possível projetar resultados desejáveis, sem o que, por sua vez, não há referência para escolher intervenções pertinentes.

A avaliação, entendida como a obtenção de dados de exame físico, juntamente com dados de entrevista (informações sobre problemas prévios de saúde, o que está acontecendo na vida do paciente, pontos fortes que ele poderia ter, medicamentos que usa, história cirúrgica, entre outras), é absolutamente essencial para o diagnóstico de enfermagem.

Infelizmente, como acontece com o processo de enfermagem como um todo, muitos enfermeiros veem a avaliação como um "formulário para preencher" ou uma série de telas de computador que têm de ser preenchidas, sem usar esses dados para raciocinar, tomar decisões e planejar o cuidado para cada paciente.

Além de algumas vezes ser vista apenas como uma obrigação a cumprir, a avaliação do paciente nem sempre é estruturada a partir da perspectiva da enfermagem como disciplina. As bases da avaliação incluem o conhecimento do raciocínio clínico e o conhecimento da disciplina de enfermagem. A avaliação requer pensamento reflexivo para permitir a visualização de padrões que emergem dos dados. Para identificar padrões é necessário a integração do conhecimento dos conceitos de enfermagem que o profissional tem com as informações que obtém sobre o paciente. $\mathrm{O}$ desenvolvimento do conhecimento sobre os conceitos da disciplina de enfermagem torna-se, então, um requisito essencial para o processo de avaliação. Por exemplo, quando nós consideramos o diagnóstico de troca de gases prejudicada, devemos ter a compreensão dos conceitos de ventilação/respiração. Exemplos de alguns dos principais conceitos de conhecimento de enfermagem subjacentes incluem: balanço energético, sono, tolerância atividade, mobilidade física e cognição. Estes conceitos são conhecimentos básicos que fundamentam diagnósticos de enfermagem tais como: fadiga, insônia, privação de sono, intolerância à atividade e confusão aguda.

Os enfermeiros precisam ter conhecimentos dos conceitos que fundamentam os diagnósticos que estão considerando e que no final estabelecem. É com base nesse conhecimento que o profissional define o que avaliar. $\mathrm{O}$ foco da resposta do paciente (por exemplo, a mobilidade física), junto com o julgamento do enfermeiro sobre o foco (por exemplo, prejudicada) forma o diagnóstico de enfermagem (mobilidade física prejudicada). O julgamento do enfermeiro (isto é, o descritor ou modificador, a opinião clínica) refina o significado do foco diagnóstico. Os SLP apoiam a compreensão do enfermeiro sobre esses conceitos, fornecendo definições sobre os mesmos, junto com os critérios de avaliação (etiologias, fatores de risCo, sinais / sintomas) e indicadores de resultados específicos e mensuráveis que são usados para avaliar a efetividade de intervenções de enfermagem específicas.

Sem uma sólida compreensão dos conceitos da disciplina de enfermagem, o enfermeiro tem lacunas de conhecimento que não lhe permitem diagnosticar com acurácia as respostas de seus pacientes aos problemas de saúde ou aos processos de vida. Sem diagnósticos acurados, o desenvolvimento de planos de tratamento adequados fica limitado, assim como as possíveis contribuições da enfermagem à saúde das pessoas.

\section{Linguagens padronizadas e comunicação clínica}

No âmbito da comunicação clínica as contribuições do uso de classificações são mais evidentes.

A comunicação clínica de boa qualidade é fundamental para a segurança do paciente e do profissional. Para ser de 
boa qualidade as informações documentadas precisam ser acuradas, relevantes, confidenciais, confiáveis, válidas, completas e seguras $^{(37-39)}$. O grande desafio atual é que, além de garantir todas essas características das informações documentadas, o processo de documentação tem que preservar o tempo do profissional para ações diretamente relacionados aos resultados dos pacientes. Estudos indicam de $25 \%{ }^{(40)}$ a $36 \%{ }^{(41)}$ do tempo da enfermagem é despendido em documentação.

A política de documentação clínica na enfermagem brasileira é bem definida pela Resolução COFEN No 429/2012 que "Dispõe sobre o registro das ações profissionais no prontuário do paciente, e em outros documentos próprios da enfermagem, independente do meio de suporte - tradicional ou eletrônico"(42). É obrigatório que sejam registrados no prontuário do paciente, além dos dados de avaliação, os diagnósticos, intervenções e resultados de enfermagem.

Para atender a essa exigência e garantir o mínimo de dispêndio de tempo na documentação e a qualidade dos registros, o uso de sistemas de linguagens padronizadas para estruturar a documentação clínica é altamente recomendado. Resultados de pesquisas mostram que documentação estruturada melhora a completude da documentação de enfermagem $^{(43-45)}$, favorece a continuidade do cuidado ${ }^{(46)}$, e que a implementação de sistemas de linguagens padronizadas tem benefícios para a assistência de enfermagem, com a melhora da comunicação clínica e organização dos serviço ${ }^{(47)}$.

\section{Linguagens padronizadas e gerenciamento clínico}

No âmbito do gerenciamento clínico o uso de classificações contribui para explicitar as decisões clínicas envolvidas na qualidade, segurança e resultados do cuidado para o paciente e família.

Na perspectiva mais ampla da administração em enfermagem, os sistemas de linguagens padronizadas têm papel importante na provisão informações necessárias para a avaliação de resultados ${ }^{(37)}$, incluindo as análises de custos e de efetividade clínica ${ }^{(37,48)}$. A avaliação de resultados dos serviços de saúde depende da disponibilidade de dados que possam ser comparados. Para que possam ser comparados é necessário que possam ser recuperados, integrados e sintetizados o que não é possível sem o uso de sistemas de linguagens padronizadas ${ }^{(49)}$.

\section{CONSIDERAÇÕES FINAIS}

Desde a década de 70 a literatura tem apontado as contribuições que os SLP podem agregar na construção do conhecimento da disciplina, no raciocínio e na prática clínica de enfermagem. Os SLP, mais do que proporcionar um método claro para a documentação, fornecem orientação e apoio para o enfermeiro em seu raciocínio clínico e nomeia os fenômenos de interesse da disciplina de enfermagem. Todo enfermeiro sabe a definição de infarto do miocárdio e como ele é diagnosticado (sinais / sintomas) - mas esse é um diagnóstico médico. Todo enfermeiro deve saber a definição de troca de gases prejudicada e como ela é diagnosticada (sinais / sintomas) - um diagnóstico de enfermagem.

O uso informado, criterioso e legítimo dos SLP requer que a ênfase seja depositada no seu verdadeiro propósito que é o de nomear os fenômenos de interesse da disciplina de enfermagem.

\section{REFERÊNCIAS}

1. Amendoeira J. Ensino de enfermagem: perspectivas de desenvolvimento. Pensar Enferm 2009;13(1):2-13.

2. Rutherford MA. Standardized nursing language: what does it mean for nursing practice? J Issues Nurs [periódico na internet]. 2008 [acesso em 4 ago 2013];13(1). Disponível em: http://www.nursingworld.org/MainMenuCategories/ThePracticeofProfessionalNursing/Health-IT/ StandardizedNursingLanguage. html?css = print

3. Norris CM. Concept clarification in nursing. Rockville: Aspen; 1982.

4. Café L, Brascher M. Organização da informação ou organização do conhecimento? In: Encontro Nacional de Pesquisa em Ciência da Informação [evento na internet]. 2008 ago 28; São Paulo, Brasil [acesso em 4 ago 2013]. Disponível em: http://www.ancib.org.br/media/dissertacao/1835.pdf.

5. Meleis Al. Theoretical nursing: development and progress. 5. ed. Philadephia: Lippincott William \& Wilkins; 2012.

6. Baldwin DA. The concept of security. Review International Studies 1997;23:5-26.

7. Mendonça ND. O uso dos conceitos: uma tentativa de interdisciplinaridade. 2nd Ed. Petrópolis: Vozes; 1985.
8. Fawcett J, Desanto-Madeya S. The structure of contemporary nursing knowledge. In: Fawcett J, Desanto-Madeya $\mathrm{S}$, editoree. Contemporary nursing knowledge - analysis and evaluation of nursing models and theories. Philadelphia: Davis; 2013. p. 3-25.

9. Chaves ECL, Carvalho EC, Goyatá SL, Galvão CM. Spiritual distress: integrative review of literature. OBJN [periódico na internet]. 2008 [acesso em 4 ago 2013];7(2). Disponível em: < http://www.objnursing.uff.br/index.php/ nursing/article/view/j.1676-4285.2008.1551>.

10. Gruber TR. A translation approach to portable ontology specifications. Knowl Acquis 1993; 5(2):199-220.

11. Guarino N. Formal Ontology in Information Systems. In: Anais de FOIS'98 [evento na internet]. 1998 jun 6-8; Trento, Itália [acesso em 4 ago 2013]. Disponível em: http://www.mif.vu.lt/ donatas/Vadovavimas/Temos/OntologiskaiTeisingasKoncepcinisModeliavimas/papildoma/ Guarino98-Formal\% 20Ontology\% 20and \% 20Information $\% 20$ Systems.pdf

12. Maran V, Augustin I, Librelotto GR, Saccol DB. Uma definição ontológico de elementos e contexto relevantes na adaptação de documentos em ambientes hospitalares pervasivos. Rev Bras Computação Aplicada 2013;5(1):26-41. 
13. Vital LP, Café LMA. Ontologias e taxonomias: diferenças. Perspect Ciênc Info 2011;16(2):115-130.

14. Biblioteca Terra Forum consultores [homepage na internet]. Taxonomia: elemento fundamental para a gestão do conhecimento [acesso em 4 ago 2013]. Disponível em: http:// biblioteca.terraforum.com.br/BibliotecaArtigo/libdoc0000 0102v003taxonomia_\%20fundamental_GC.pdf

15. Von Krogh G. An examination of the NANDA International Taxonomy for domain completeness, ontological homogeneity and clinical functionality. Int J Nurs Terminol Classif 2008;19(2):65-75.

16. Risjord M. Rethinking concept analysis. J Adv Nurs 2009;65(3)684-91.

17. Morse J. Exploring theoretical basis of nursing advanced techniques of concept analyses. Adv Nurs Sci 1995; 17(3):31-46.

18. Whitley GG. Processes and methodologies for research validation of nursing diagnoses. Nurs Diagn 1999;10(1):5-14.

19. Walker LO, Avant KC. Strategies for theory construction in nursing. $5^{\text {th }}$ ed. New Jersey: Prentice Hall; 2010.

20. Mota DDCF, Cruz DALM, Pimenta CAM. Fadiga: uma análise do conceito. Acta Paul Enferm 2005; 18(3):285-93.

21. Araújo GBS, Garcia TR. Adesão ao tratamento anti-hipertensivo: uma análise conceitual. Rev Eletrônica Enferm [periódico na internet]. 2006;8(2):259-72

22. Santos ER, Souza MF, Gutiérrez MGR, Maria VLR, Barros ALBL. Validação do conceito risco de débito cardíaco diminuído. Rev Latino-Am Enferm 2013;21(n. esp.):97-104.

23. Appoloni $\mathrm{AH}$, Herdman $\mathrm{TH}$, Napoleão AA, Carvalho EC, Hortense P. Concept analysis and validation of the nursing diagnosis, delayed surgical recovery. Int J Nurs Knowl 2013.

24. Rodgers BL, Knafl KA. Concept development in nursing: foundations, techniques, and applications. $2^{\text {nd }}$ ed . Philadelphia: Saunders; 2000.

25. Ramanzini AE. Recuperação cirúrgica retardada: análise do conceito. Ribeirão Preto. Dissertação [Mestrado em Enfermagem] - EERP-USP; 2013.

26. Pettengil MAM. Vulnerabilidade da família: desenvolvimento do conceito. São Paulo. Tese [Doutorado em Enfermagem] - EERP-USP; 2003.

27. Poles K. O desenvolvimento do conceito morte digna na UTI pediátrica. São Paulo. Tese [Doutorado em Enfermagem] - EERP-USP; 2008.

28. Scroggins LM. The developmental processes for NANDA International Nursing Diagnoses. Int J Nurs Terminol Classif 2008;19(2):57-63.

29. Moorhead S, Johnson M, Maas ML, Swanson E. Nursing outcomes classification (NOC). $5^{\text {th }}$ ed. Saint Louis: Elsevier; 2013.

30. Bulecheck GM, Butcher HK, Dochterman JM, Wagner C. Nursing interventions classification (NIC). $6^{\text {th }}$ ed. Saint Louis: Elsevier; 2013.

31. Herdman TH, Von Krog G. A taxonomia II da NANDA International 2012-2014. In: NANDA International.
Diagnósticos de enfermagem da NANDA: definições e classificação 2012-2014. Porto Alegre: Artmed; 2013. p. 91-106.

32. Bordage G, Grant J, Marsden J. Quantitative Assessment of diagnostic ability. Med Educ 1990;34:413-25.

33. Gale J, Marsden P. Clinical problem solving: the beginning of the process. Med Educ 1982;16(1):22-26.

34. Grant J, Marsden P. The structure of memorized knowledge in students and clinicians: an explanation for diagnostic expertise. Med Educ 1987;21:92-8.

35. Fontes $\mathrm{CMB}$. Perfil de diagnósticos de enfermagem antes e após a implementação da classificação da NANDA-I. São Paulo. Tese [Doutorado em Enfermagem]- EEUSP; 2006.

36. Vóvio CL. Duas modalidade de pensamento: pensamento narrativo e pensamento lógico-científico. In Oliveira MB, Oliveira MK, organizadores. Investigações cognitivas: conceitos, linguagem e cultura. Porto Alegre: Artes Médicas Sul; 1999

37. Cusack CM, Hripcsak $G$, Bloomrosen $M$, Rosenbloom ST, Weaver CA, Wright A, et al. The future state of clinical data capture and documentation: a report from AMIA's 2011 Policy Meeting. J Am Med Inform Assoc 2013;20(1):134-40.

38. Keenan G, Yakel E, Dunn-Lopez K, Tschannen D, Ford YB. Challenges to nurse's efforts of retrieving, documenting, and communicating patient care information. J Am Med Inform Assoc 2013;20(2):245-51.

39. Wang N, Hailey D, Yu P. Quality of nursing documentation and approaches to its evaluation: A mixed-method systematic review. J Adv Nurs 2011;67:1858-1875.

40. Lomas C. The burden of bureaucracy. Nurs Stand 2012;26(30):22-24.

41. Hendrich A, Chow MP, Skierczynski BA, Lu Z. A 36-hospital time and motion study: how do medical-surgical nurses spend their time? Perm J 2008;12(3):24-34.

42. Conselho Federal de Enfermagem (Brasil). Resolução $n^{\circ}$. 429 , de 30 de maio de 2012. Dispõe sobre o registro das ações profissionais no prontuário do paciente, e em outros documentos próprios da enfermagem, independente do meio de suporte - tradicional ou eletrônico. Diário Oficial da União 8 jun 2012;Seção 1.

43. Whited K, Aiyagari V, Calderon-Arnulphi M, Cursio J, Pandey D, Hillmann $M$, et al. Standardized admission and discharge templates to improve documentation of The Joint Commission on Accreditation of Healthcare Organization performance markers. J Neurosci Nurs 2010;42(4):225-8.

44. Darmer MR, Ankersen L, Nielsen BG, Landberger G, Lippert E, Egerod I. Nursing documentation audit - the effect of a VIPS implementation programme in Denmark. J Clin Nurs 2006;15:525-534.

45. Daly JM, Buckwalter K, Maas M. Written and computerized care plans: organizational process and effect on patient outcomes. J Gerontol Nurs 2002;28:14-23.

46. Keenan G, Yakel E. Promoting safe nursing care bringing visibility to the disciplinary aspects of interdisciplinary care. AMIA Annu Symp Proc 2005;385-9. 
47. Furuya RK, Nakamura FRY, Gastaldi AB, Rossi LA. Sistemas de classificação de enfermagem e sua aplicação na assistência: revisão integrativa de literatura. Rev Gaúch Enferm 2011;32(1):167-75.

48. Stone PW, LeeJ, Giannini M, Bakken S. Economic evaluations and usefulness of standardized nursing terminologies. Int J Nurs Terminol Classifications 2004;15(4):101-113.

49. Elfrink V, Bakken S, Coenen A, NcNeil B, Bickford C. Nursing vocabularies: a foundation for quality care. Sem Oncol Nur 2001;(17)1:18-23. 